This is the peer reviewed version of the following article: Hassanin, Hany, Alkendi, Yusra, Elsayed, Mahmoud, Essa, Khamis and Zweiri, Yahya (2020) Controlling the properties of additively manufactured cellular structures using machine learning approaches. Advanced Engineering Materials, 22(3), p. 1901338., which has been published in final form at https://doi.org/10.1002/adem.201901338. This article may be used for noncommercial purposes in accordance with Wiley Terms and Conditions for Use of Self-Archived Versions. 


\title{
Controlling the Properties of Additively Manufactured Cellular Structures using Machine Learning Approaches
}

Hany Hassanin $^{\text {I* }}$ Yusra Alkendi ${ }^{2}$, Mahmoud Elsayed $^{3}$, Khamis Essa ${ }^{4}$, and Yahya Zweiri ${ }^{5,2}$ 1 School of Engineering, University of Liverpool, Liverpool, L69 3BX, UK

2 Khalifa University Center for Autonomous Robotic Systems, Department of Aerospace Engineering, Khalifa University of Science and Technology, P.O. Box 127788 Abu Dhabi, $U A E$

3 Department of Industrial and Management Engineering, Arab Academy for Science and Technology and Maritime Transport, PO Box 1029, Abu Qir, Alexandria 21599, Egypt 4 University of Birmingham, Edgbaston, B15 2TT, UK

5 Faculty of Science, Engineering and Computing, Kingston University London, London SW15 3DW, UK

Corresponding author: Hany Hassanin h.hassanin@liverpool.com

The authors declare no conflict of interest

Keywords: $L P B F$; lattices; deep learning;

\begin{abstract}
Cellular structures are lightweight-engineered materials that have gained much attention with the development of additive manufacturing technologies. This paper introduces a precise approach to predict the mechanical properties of additively manufactured lattice structures using deep learning approaches. Diamond shaped nodal lattice structures were designed by varying strut length, strut diameter and strut orientation angle. The samples were manufactured using laser powder bed fusion (LPBF) of Ti-64 alloy and subjected to compression testing to measure the ultimate strength, elastic modulus, and specific strength. Machine learning approaches such as shallow neural network (SNN), deep neural network (DNN), and deep learning neural network (DLNN) were developed and compared to the statistical design of experiment (DoE) approach. The trained DLNN model showed the highest performance when compared to DNN, DoE and SNN with a mean percentage error of 5.26\%, 14.60\%, and 9.39\% for the ultimate strength, elastic modulus, and specific strength, respectively. The DLNN model was used to create process maps, and was further validated. The results showed that although deep learning is preferred for big data, the optimised DLNN model outperformed the statistical DoE approach and can be a favourable tool for lattice structure prediction with limited data.
\end{abstract}




\section{Introduction}

Metal cellular structures are high performance lightweight-engineered materials, which have combination of high load bearing strength, high-energy absorption, and unique acoustic and thermal insulation properties. These properties made them promising structures for high performance products such as filters, catalytic convertors, acoustic absorbers, heat exchangers, abradable seals, porous burners, biomedical implants, and oil sensors [1-5]. The structures can be categorised into periodic and stochastic porous structures. Pores of the periodic lattice structures are uniform as they were made from repeated unit cells, whereas they are randomly distributed in the stochastic porous structures. Generally, the mechanical properties of periodic lattice structures outperform those exhibits by the stochastic porous structures because of their internal imperfections. However, the complexity and time consuming of manufacturing periodic lattice structures using conventional manufacturing technologies such as casting and machining obstruct the wide use of these meta-materials $[6,7]$.

Additive manufacturing (AM) has been widely explored as a robust technology for the fabrication of complex geometrical structures reducing the manufacturing steps and constraints of conventional manufacturing technologies [8-10]. Laser powder bed fusion (LPBF) is a metal AM technique, which builds up 3D components by using a laser beam to selectively melt layers of metal powder according to a digital design. The technique is capable to rapidly manufacture high-resolution cellular lattice structures for many applications. Several researches have been introduced to investigate the manufacturing of periodic cellular lattice structures using LPBF techniques. Process optimisation is an efficient approach to control and optimise the performance of periodic lattice structures. Typically, LPBF process optimisation investigates the effect of parameters such as laser power, laser scanning speed, hatching spacing, build temperature, layer thickness and scanning strategy on the properties of the developed structures such as porosity, cracks, defects and microstructure [11-15]. Li et al. studied the development of shape memory auxetic cellular structures and investigated the role of LPBF process parameters on the as fabricated and heat treated microstructure and hence the performance of the developed structures [16]. On the other hand, Yan et al. studied the manufacturability and mechanical properties of novel Schoen Gyroid lattice structures using LPBF [17]. The study also investigated the effect of the cell size on the yield strength and the Young's modulus of the developed lattice structures. McKown et al. studied the compression behaviour of several metallic lattice designs based on octahedral and pillar-octahedral geometries using 
mathematical, numerical, and density mapping to control the properties of the developed structures [18-20]. The above simulations are effective but they focus on one or few aspects of the design process and it becomes difficult to predict the performance of additively manufactured periodic lattice structures accurately via simulation approaches only.

Recently machine learning (ML) has been shown to accelerate the development of AM technology and enable new opportunities in many applications [21]. The benefits of this technology is that they do not need to develop analytical equations but they learn the relation between the input or design parameters and the output performance based on existing data. Neural network $(\mathrm{NN})$ is a supervised ML technique that can be trained to show a particular performance. An input layer, a hidden layer, and an output layer are the three types of layers included in any NN architecture. Each layer consists of a number of neurons or nodes. In NN, an arbitrary function can be expressed network using a sufficient number of neurons and at least one hidden layer. A shallow neural network $(\mathrm{SNN})$ is a type of $\mathrm{NN}$ with an input layer, one or two hidden layers, and one output layer. Deep learning neural networks (DLNN) is a part of machine-learning family that utilise several hidden layers in their architecture to learn how data is presented, which allows automated search of big data. Coefficients in NN, also known as weights, represent the interaction between neurons in neighbouring layers, are obtained by training the $\mathrm{NN}$ iteratively aiming to minimize the discrepancy between predicted and actual outputs. Typically, DLNNs outperforms SNNs with respect to the number of computational units, particularly for complex problem. This is because of the non-linear nature the activation functions taking place at several layers in the DLNN network [22]. On the other hand, training deep networks can be challenging when the initial weights are close to the optimum values. Furthermore, if the initial weights are far from the optimum values, either greater or less, the training process will approach a local minima or will be infeasible [23]. Accordingly, the potential of deep learning research remained unexplored widely until the introduction of the greedy layer-wise pre-training method [23, 24], where a restricted Boltzman machine was used to train a deep belief network. Later, a stacked auto-encoder (SAE) architecture was proposed by Bengio et al. [22] to replace the restricted Boltzman machine in an analogous pre-training method. Greedy layer-wise pre-training initializes the neural network' weights to values close to a local minimum. As a result, it helps the optimization process and produces efficient model generalization [22]. The term greedy refers to pre-training every layer individually, irrespective of subsequent layers in the network [22]. Accordingly, 
each layer is provided with data at a separate abstraction level and learn to represent the data in a distinct manner [24]. One case study was found to use machine learning in the design of hierarchical materials of AM parts [25]. The authors investigated optimized properties of hierarchical materials based on a trained model of finite element analysis database of hundreds of thousands of geometries without the use of full microstructural data. However, the study did not provide details about the AM technique, used material, or process parameters. In this work, we use machine-learning approaches to predict the properties of additively manufactured titanium alloy cellular structures and hence optimize their properties. The cell size and orientation were considered as input parameters. The ultimate compression strength, Young' modulus, and specific strength were studied as output data. On the other hand, statistical and machine learning approaches such as DoE, SNN, DNN, and DLNN were used to analytically understand the effect of the input design parameters on the performance of AM cellular structures processed by LPBF.

\section{Cellular Structure Design}

Periodic cellular structures with defined geometries exhibit controlled density to achieve specific properties [26]. Design parameters of periodic lattice structures include density, pores size, and features dimensions. There are many types of periodic lattice structures such as Gyroid, Diamond, and Neovius [27]. They are different in their strut shape, orientation, thickness, and nodal connectivity. Among these types, the diamond shape lattice structure is considered as a favourable structure and hence has been used in many applications [28, 29]. Diamond shape lattice structure has four struts connected nodally with another four struts, which allows a great flexibility in changing the volume fraction and without the use of support structures. Figure 1 shows the nodally-connected diamond lattice structure and its unit cell. The investigated design parameters of this structure used in this study are the strut length (L), strut diameter (D), and the strut orientation angle theta $(\theta)$. The generated design parameters and levels are shown in Table 1 . The levels values listed in Table 1 were chosen based on a combination of the manufacturability of the samples and the geometrical constraints to achieve compression samples according to ASTM C365. A design of experiment technique (DoE) using response surface is used in this paper as a reference approach. 
(a)

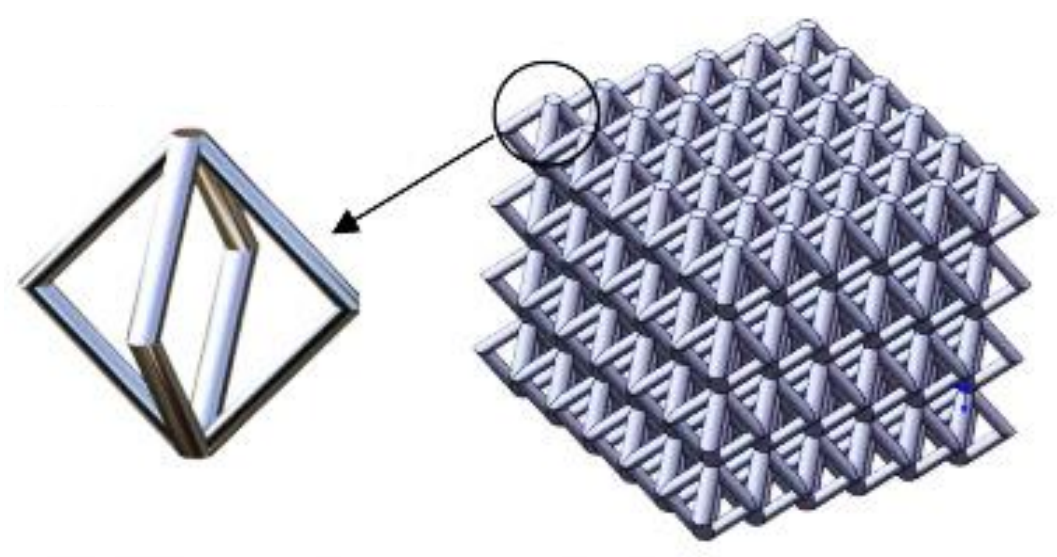

(b)

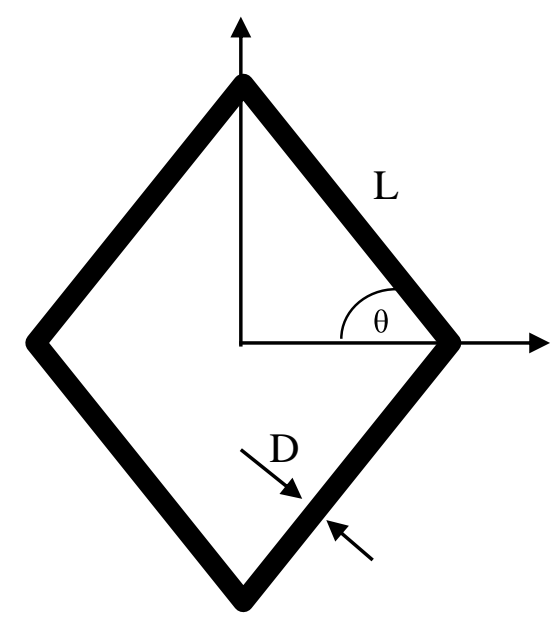

Figure 1: The Nodally connected diamond structure used in this study and their design parameters.

Table 1: Design parameters and their corresponding levels

\begin{tabular}{lllllll}
\hline Parameter & Units & \multicolumn{5}{c}{ Levels } \\
\cline { 3 - 7 } & & $\mathbf{- 2}$ & $\mathbf{- 1}$ & $\mathbf{0}$ & $\mathbf{1}$ & $\mathbf{2}$ \\
\hline Strut Length (D) & $\mathrm{mm}$ & 0.2 & 0.36 & 0.6 & 0.83 & 1 \\
\hline Strut diameter (L) & $\mathrm{mm}$ & 1 & 1.2 & 2.25 & 3.29 & 4 \\
\hline Strut Orientation Angle $(\theta)$ & degree & 30 & 36.1 & 45 & 53.9 & 60 \\
\hline
\end{tabular}

\section{Deep Learning}

In this section, the structure of the proposed deep learning neural network (DLNN), in terms of its depth, size of hidden layers, and its activation functions, is presented. Then, the adopted 
pre-training and fine-tuning techniques are demonstrated. Finally, backpropagation algorithm is discussed. The shallow neural network ( $\mathrm{SNN}$ ), shown in Figure 2, is presented and used for comparison purpose to evaluate the performance of the developed DLNN.

Proposed DLNN Architecture: The structure of the developed deep learning neural network (DLNN), shown in Figure 3, gave the best performance among many other tested structures having different hidden layers, activation functions, and neurons sizes. It comprises of an input layer, three hidden layers, and an output layer. The input layer has three inputs, the struts length, diameter and orientation angle. The output layer consists of three nodes, activated by the sigmoid function. The outputs of the network are the ultimate strength, Young' modulus, and specific strength. The three hidden layers have the same size, 50 neurons in each layer. Employing hidden layers of the same size was recommended in [24] due to its convenience when applying the pre-training technique that will be presented shortly. As for the activation functions, Swish was chosen to activate the first hidden layer, followed by a rectified linear unit (ReLU) which activates the second and third hidden layers as expressed in equations (2) and (3), respectively.

$$
\begin{aligned}
& \mathrm{f}_{1}(\mathrm{x})=\mathrm{x} \cdot \operatorname{sigmoid}(\mathrm{x})=\frac{\mathrm{x}}{1+\mathrm{e}^{-\mathrm{x}}} \\
& \mathrm{f}_{2}(\mathrm{x})=\max (0, \mathrm{x})
\end{aligned}
$$

\section{DLNN Pre-training:}

Since the performance of a neural network heavily relies on the assigned weights [30], it is essential to guarantee having an initial weights which are in the neighborhood of a good estimate so as to ensure that the employed gradient descent method converges to the local minimum area during training without have the vanishing gradient issue[22, 23]. To this end, a pre-training technique is developed in the proposed approach to initialize the network's weights. The selected pre-training technique is the developed unsupervised greedy layer-wise and its process is depicted in Figure 4. The proposed DLNN is pre-trained in four stages, where the DLNN's non-input layers are trained sequentially, starting from the first hidden layer till the output layer. Each layer is individually trained using a Shallow Neural Network (SNN), which is also denoted to as an auto-encoder, of suitable input and output layers. Only $90 \%$ of the measured data were randomly considered in the training process.

DLNN Fine-tuning: Although pre-training better initializes the weights of the DLNN, it 
suffers from sub-optimality, especially for the weights in the first hidden layers [30]. Therefore, global network fine-tuning is carried out to replace stochastic weights, resulting from unsupervised learning in the pre-training process, with more deterministic ones by means of the backpropagation algorithm [23]. The optimization process in this case is much simpler compared to optimizing random initial weights and yields better generalization[22]. Backpropagation [31] is a widely used supervised learning technique for neural networks, where the differences between the DLNN's predictions and the corresponding target outputs are employed to modify the network internal weights, thus refitting the network parameters for optimal performance. In what follows, a description of the backpropagation algorithm is provided. First, the input to the network is forward propagated through the DLNN, where each layer computes its output as a function of its preceding layer's output. Then, during the backpropagation process the error between the predicted output and the target is calculated by using one or more of the cost functions (i.e., Mean Squared Error (MSE) Mean Absolute Error (MAE), or Cross Entropy (CE) loss functions) and then propagated backword to the network layers. By using the gradient decent (GD), the updated weights are calculated by multiplying the derivative of the activation function with the computed error (between network predictions and targets). Thus, using gradient decent technique along with the backpropagation algorithm, the minimum of a least square cost function would be achieved.

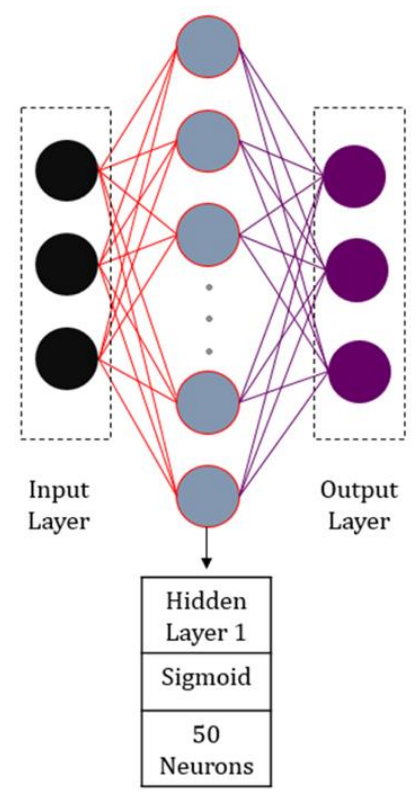

Figure 2: Shallow Neural Network Supervised Training 


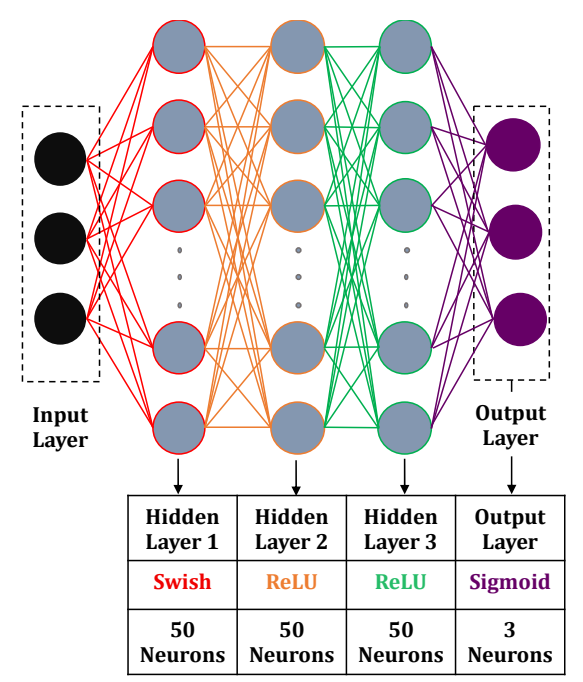

Figure 3: Deep Neural Network Supervised Training

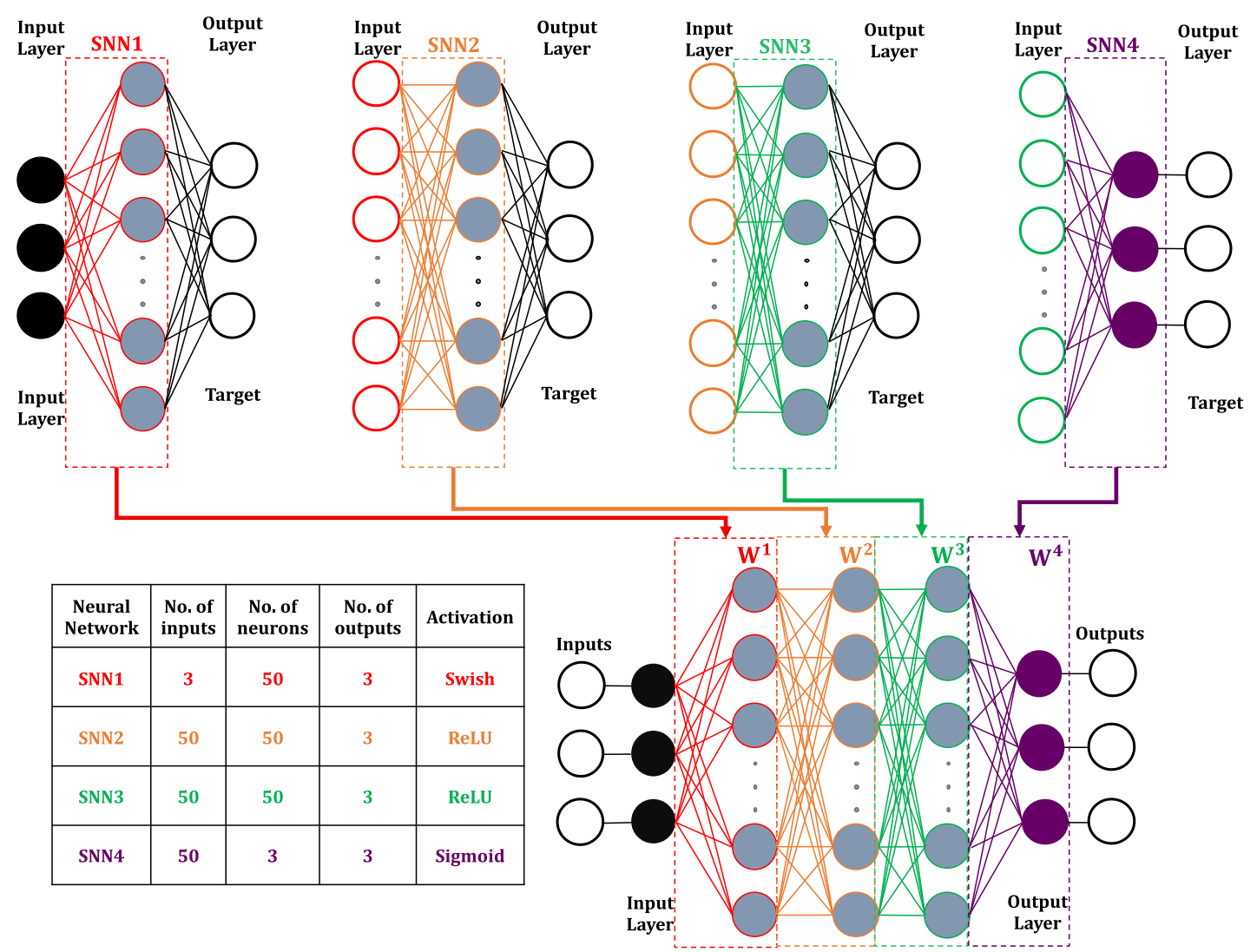

Figure 4: Deep Learning Neural Network Unsupervised Greedy Layer-wise Pre-training Approach 


\section{Results}

\subsection{Compression properties of lattice structures}

The compression setup and the properties of one of the compression test samples are shown in Figure 5. The compression stress increases to a peak value (Ultimate strength). Afterwards, the stress falls rapidly after the partial failure of some of the lattice structures nodes. The stress picks up again as the strain increases until another failure to some nodes take place. This behaviour is repeated until the whole sample fractured into two pieces. The measured properties matrix of the LPBF Ti-64 lattice structures are shown in Table 2. The table includes three design parameters (strut length, strut diameter, strut orientation angle) and the corresponding mechanical results (Ultimate strength, Specific strength and Young's modulus). The table showed that the ultimate strength of the cellular materials is in range of 4-228 $\mathrm{MPa}$, the specific strength are $12-120 \mathrm{KN} \cdot \mathrm{m} / \mathrm{kg}$, and Young's modulus 0.05-9.31 GP.

Table 2: The matrix of the investigated parameters and measured porosity and hardness

\begin{tabular}{ccccccc}
\hline Samples & $\begin{array}{c}\text { Strut } \\
\text { Length }(\mathrm{L}) \\
(\mathrm{mm})\end{array}$ & $\begin{array}{c}\text { Strut } \\
\text { diameter }(\mathrm{D}) \\
(\mathrm{mm})\end{array}$ & $\begin{array}{c}\text { Strut } \\
\text { Orientation } \\
\text { Angle }(\theta) \\
(\text { degree })\end{array}$ & $\begin{array}{c}\text { Ultimate } \\
\text { Strength } \\
(\mathrm{MPa})\end{array}$ & $\begin{array}{c}\text { Specific } \\
\text { Strength } \\
(\mathrm{KN} \cdot \mathrm{m} / \mathrm{kg})\end{array}$ & $\begin{array}{c}\text { Young's } \\
\text { modulus } \\
(\mathrm{GPa})\end{array}$ \\
\hline 1 & 2.25 & 1 & 45 & 228 & 115 & 8.34 \\
\hline 2 & 3.29 & 0.36 & 54 & 4 & 12 & 0.05 \\
\hline 3 & 3.29 & 0.84 & 54 & 19 & 25 & 0.35 \\
\hline 4 & 3.29 & 0.84 & 36 & 82 & 69 & 1.55 \\
\hline 5 & 4 & 0.6 & 45 & 7 & 17 & 0.16 \\
\hline 6 & 2.25 & 0.6 & 60 & 26 & 27 & 0.44 \\
\hline 7 & 1.21 & 0.84 & 36 & 96 & 44 & 9.31 \\
\hline 8 & 2.25 & 0.6 & 45 & 47 & 44 & 1.28 \\
\hline 9 & 3.29 & 0.36 & 36 & 9 & 22 & 0.26 \\
\hline 10 & 2.25 & 0.2 & 45 & 10 & 22 & 0.22 \\
\hline 11 & 2.25 & 0.6 & 30 & 185 & 120 & 7.73 \\
\hline 12 & 2.25 & 0.6 & 45 & 52 & 47 & 1.41 \\
\hline 13 & 1.21 & 0.84 & 54 & 69 & 43 & 4.19 \\
\hline 14 & 2.25 & 0.6 & 45 & 49 & 44 & 1.17 \\
\hline 15 & 1.21 & 0.36 & 54 & 146 & 58 & 4.95 \\
\hline
\end{tabular}


(a)

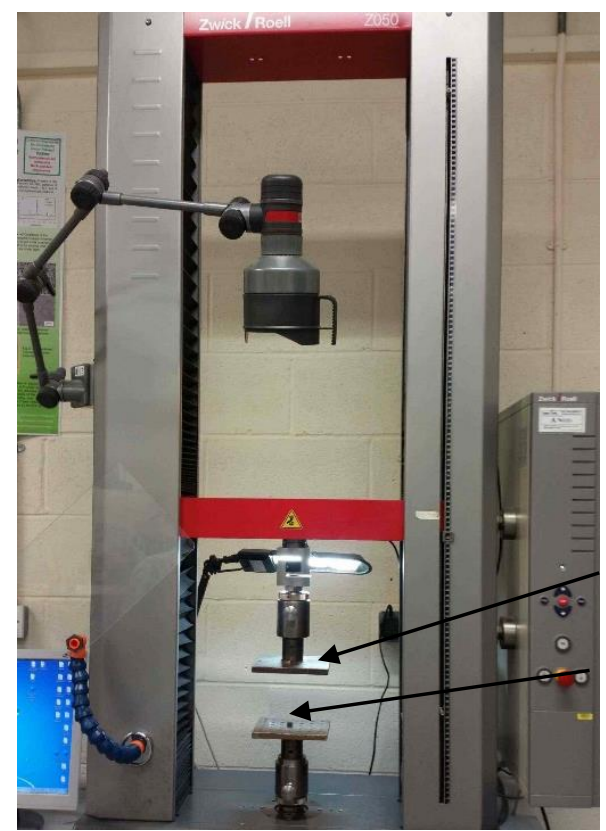

Compression

Platen

Cellular sample

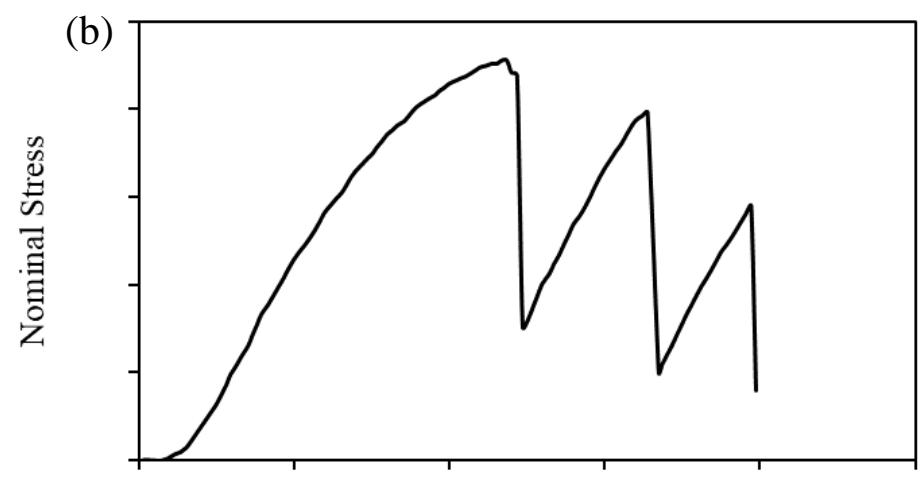

(c)

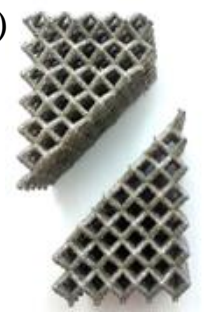

Nominal Strain

Figure 5: (a) compression testing setup, (b) one of the compression stress-strain diagram for the Ti64 cellular structures, (c) fractures sample

\subsection{Comparison between DoE and the DL Models}

After preparing the datasets, an automated search for the optimal DLNN structure was conducted by varying the initial random weights, the number of layers, the activation functions, and the number of neurons. More than 2000 different DNN structures from single to five hidden layers were trained and tested. The structure, shown in Figure 3, demonstrated the highest accuracy among all other structures, where the mean percentage error was the lowest. Comparisons between two alternatives for pre-training of the developed structure were then conducted, more specifically unsupervised greedy layer-wise pre-training, and backpropagation. The best performing model was finally compared against and proved to 
outperform other regression techniques, including deep neural network (DNN) and SNN. The DNN and SNN that were compared to the adopted DLNN were selected through an automated search, analogous to that used for DLNN, while varying the relevant parameters for each.

The performance of the proposed approach DLNN is compared to both DNN and SNN. The DNN structure was selected to be similar to the optimum DLNN structure. To select the SNN that will be compared to DLNN, several structures with various activation functions and layer sizes were tested. Figure 6 shows the corresponding prediction mean percentage error (MPE) for the different approaches Response surface, DLNN, DNN and SNN which confirms DLNN as a best performance. It's clearly shown that a SNN (the simplest structure) has the highest MPE compared to other models since it couldn't capture or correlate the hidden features or material's parameters. Thus, a more complex structure is surely essential for optimal network performance, and in this case the DLNN model.

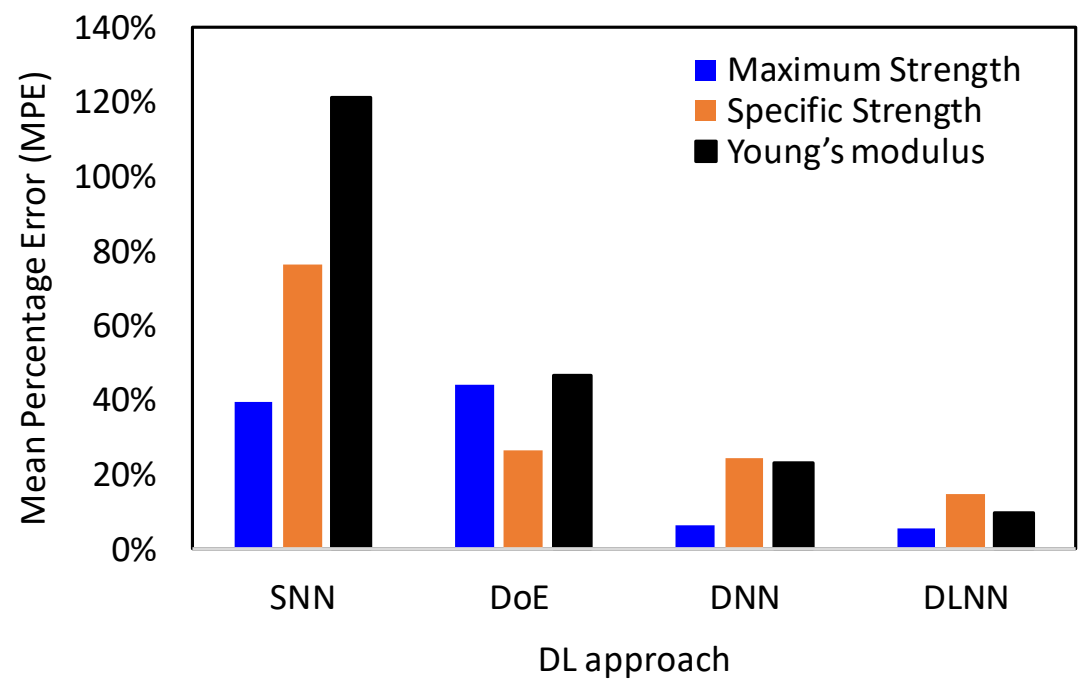

Figure 6: MPE comparison for tested approaches

\subsection{Model Validation}

In all the developed models, only $90 \%$ of the measured data were randomly considered in the search. The remaining 10\% were used to validate the model. The optimum DLNN model was validated against the experimental datasets of Ti-6246 including the 10\% unused dataset. Figure 7, Figure 8 and Figure 9 depict the porosity and hardness estimates by the developed DLNN when pre-trained using unsupervised greedy layer-wise pre-training approaches 
compared to the measured data as well as by the other two developed methods (DNN and $\mathrm{SNN}$ ). The DLNN has the ability to pre-construct a process map for the alloy hardness and densification behavior as a function of the process parameters. Table 4 lists the DLNN output predictions of porosity and hardness as a function of the design parameters for 125 number of sampling data (consists of unseen dataset (for the network) that are randomly mixed with the training dataset), to map the process boundaries.

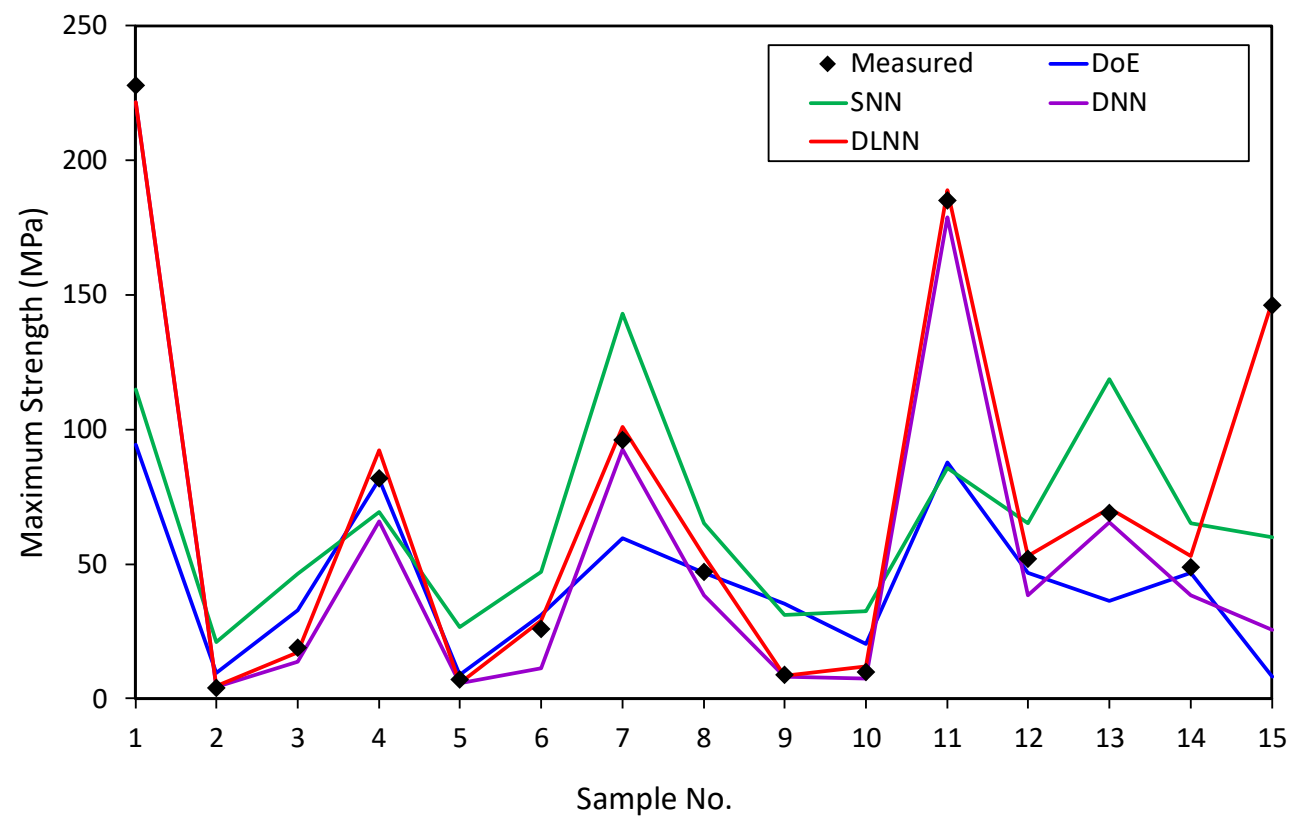

Figure 7: Maximum strength compared to the output of the DoE/DLNN/DNN/SNN models. 


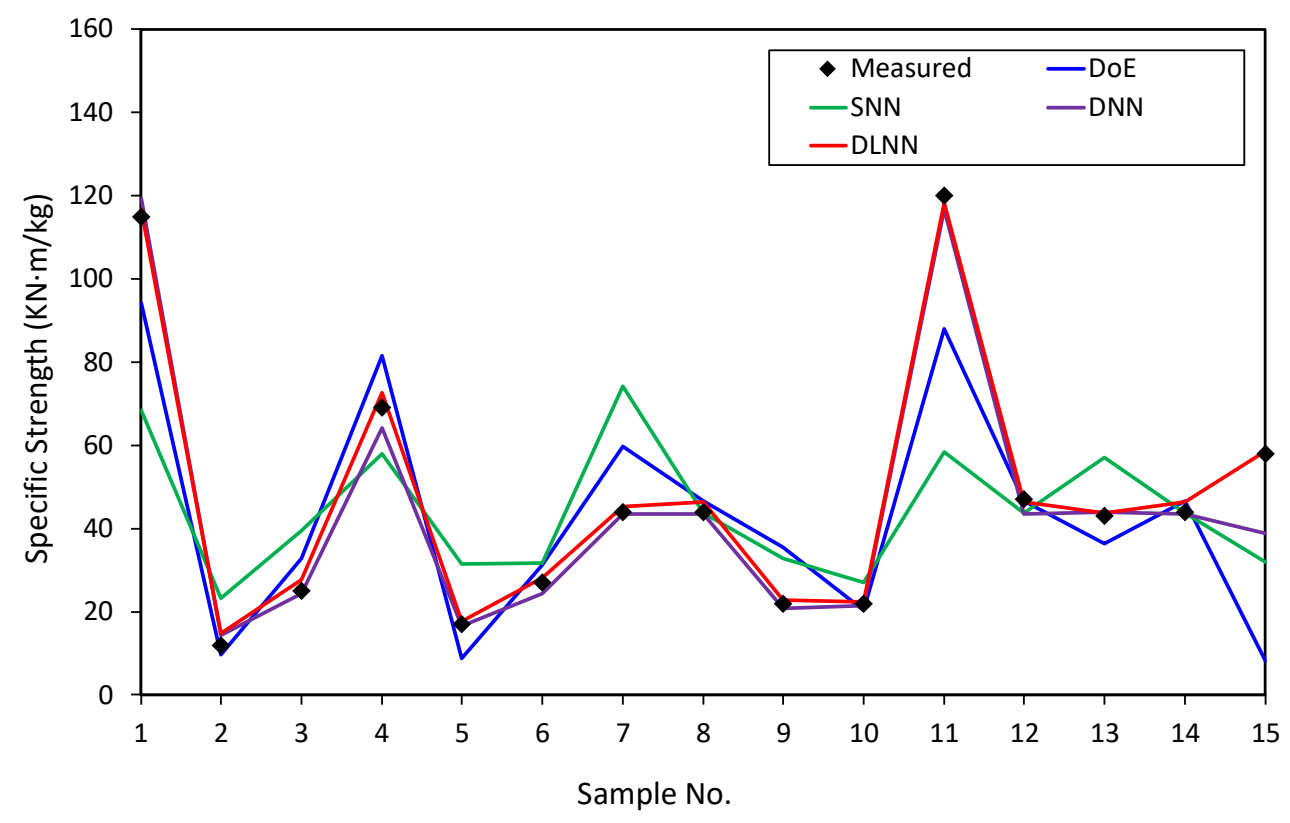

Figure 8: Specific strength compared to the output of the DLNN/DNN/DoE/SNN models

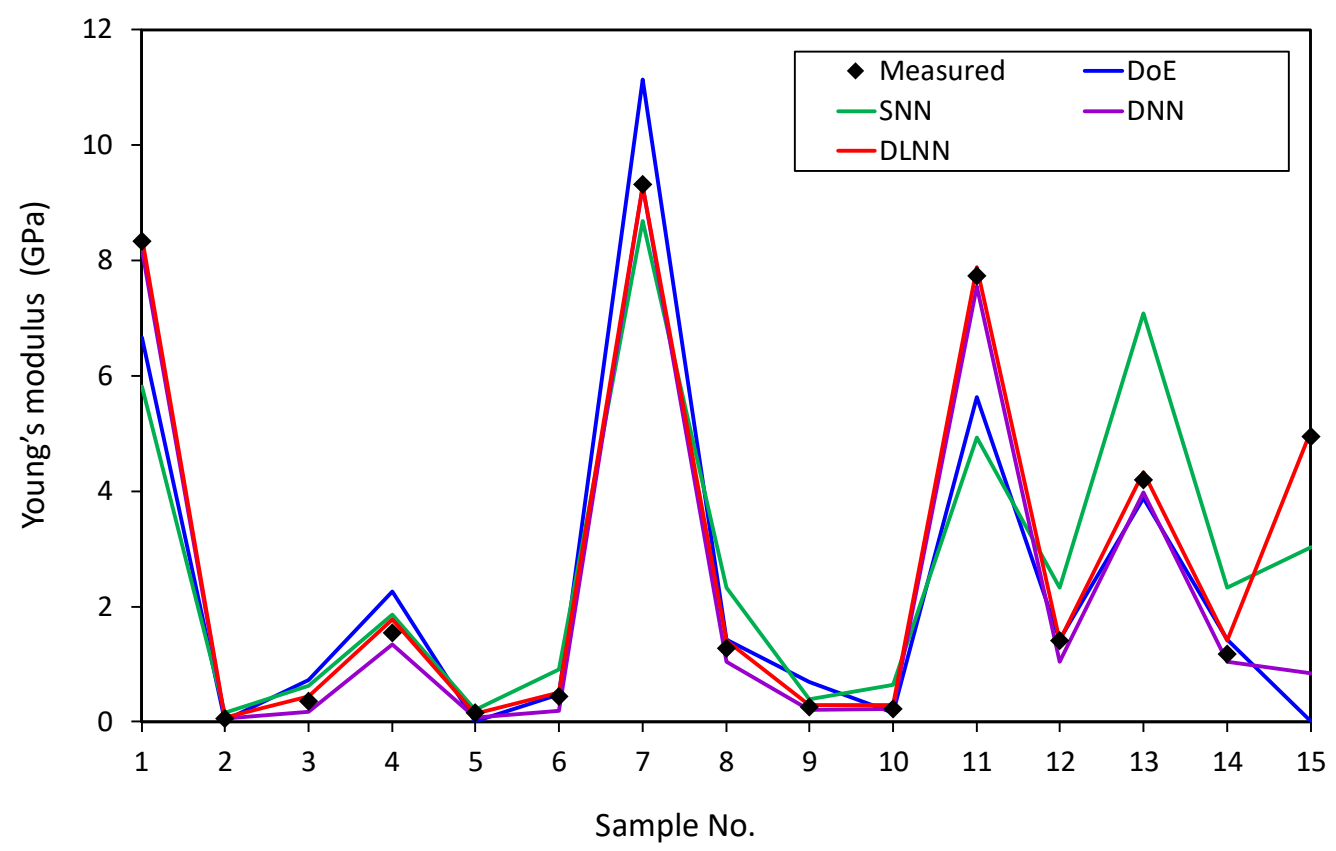

Figure 9: Young's modulus compared to the output of the DLNN/DNN/DoE/SNN models. 


\subsection{DLNN distribution map}

The optimum DLNN model was used to construct a distribution map of the compression ultimate strength, Elastic modulus and specific strength as a function of strut diameter, strut length and orientation angle. The distribution map can be used to predict the performance cellular structures as well as to study the effect of the strut diameter, strut length and strut orientation angle of the compression behavior of the samples. Figure 10 shows the contour map of the compression ultimate strength and Elastic modulus as a function strut diameter and length. In addition, the figure shows a comparison between the stress-strain diagrams of lattice samples fabricated with different strut diameter and strut length (X1-X4). X1 and X2 represent lattice samples fabricated using different strut diameter with the same strut length and orientation angle, while X3 and X4 are lattice represent samples fabricated using different strut length and the same strut diameter and orientation angle. Specifically, X1 and X2 represent lattice structures with strut length of $2.25 \mathrm{~mm}$ and strut diameter of $0.2 \mathrm{~mm}$ and $0.6 \mathrm{~mm}$, respectively. On the other hand, X3 and X4 represent lattice structures with strut diameter of $0.84 \mathrm{~mm}$ and strut length of $1.21 \mathrm{~mm}$ and $3.29 \mathrm{~mm}$, respectively. Generally, there is an increase in the compression strength as the strut diameter increases and the strut length decreases. Similarly, the elastic modulus increases with the increase of the strut diameter and the decrease strut length. 

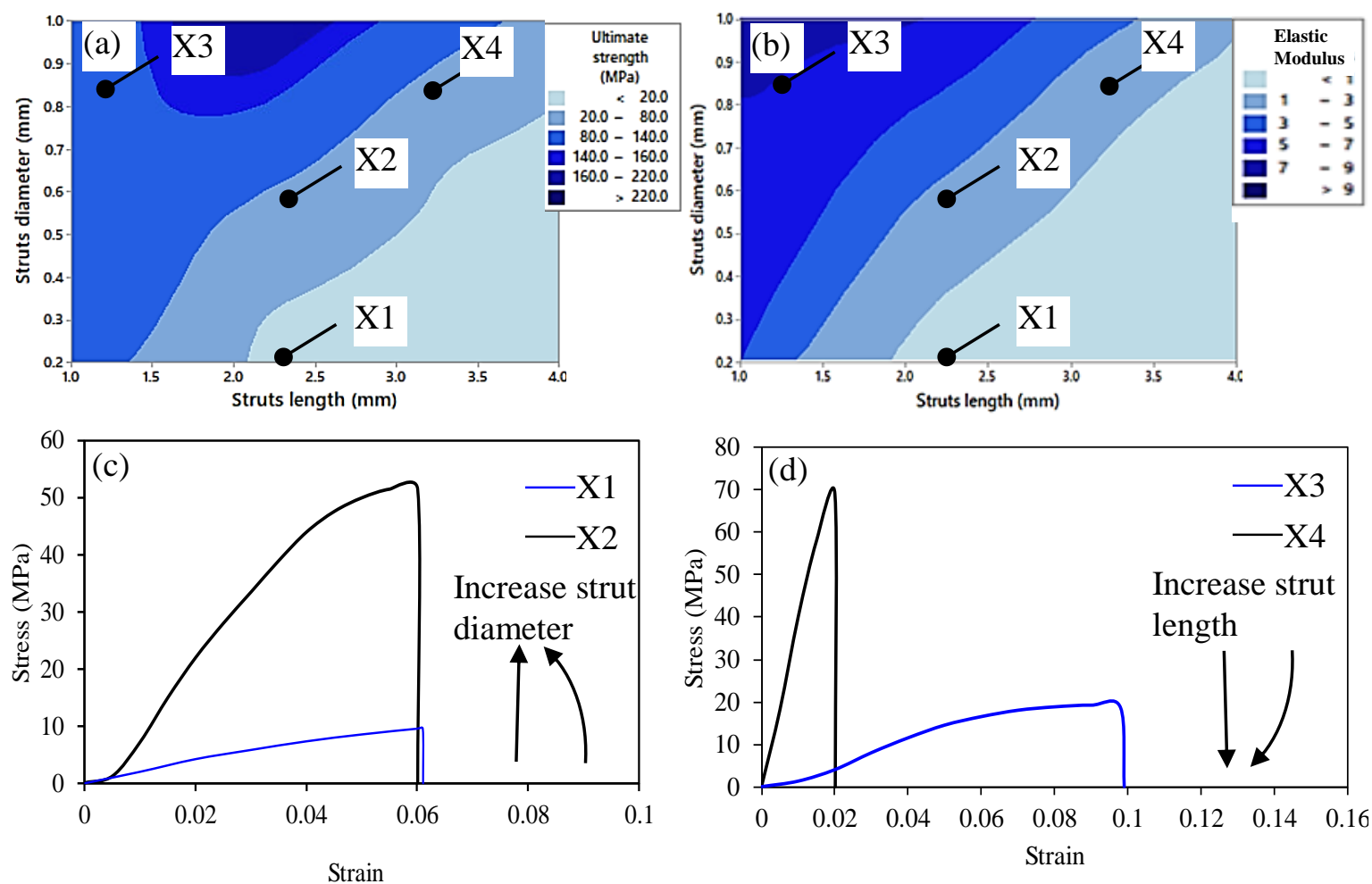

Figure 10: $(a-b)$ the predicted contours distribution of the ultimate compression strength and elastic modulus with respect to the lattice struts diameter and length (c-d) stress strain diagrams of samples X1-X4.

Figure 11 shows the contour map of the compression ultimate strength and Elastic modulus as a function strut diameter and orientation angle. Similarly, the figure compare the stress-strain diagrams of lattice samples fabricated with different strut angles (X5-X6). The figure shows a decrease in the compression strength as the strut angle increases. On the other hand, the elastic modulus decreases with the increase of the strut diameter and strut length. Figure 12 shows the predicted contours distribution of the specific strength with respect to the lattice struts diameter, length and angle. The figure shows similar behavior as in the ultimate strength prediction. The specific strength of the samples increases as the dimeter increases while it decreases with the increase of the strut length and strut angle. 

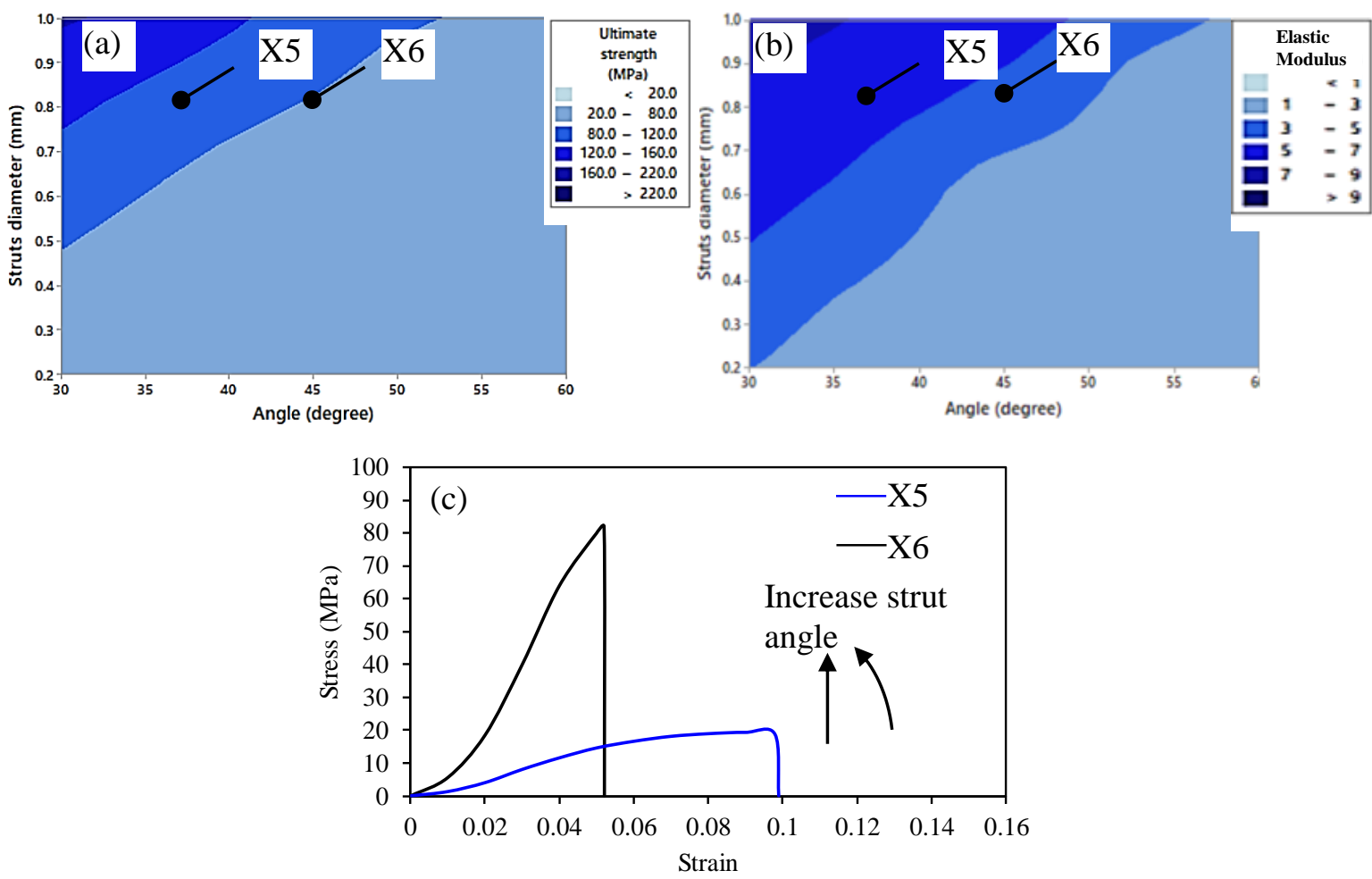

Figure 11: (a-b) the predicted contours distribution of the ultimate compression strength and elastic modulus with respect to the strut angle, (c) stress strain diagrams of samples X5-X6.
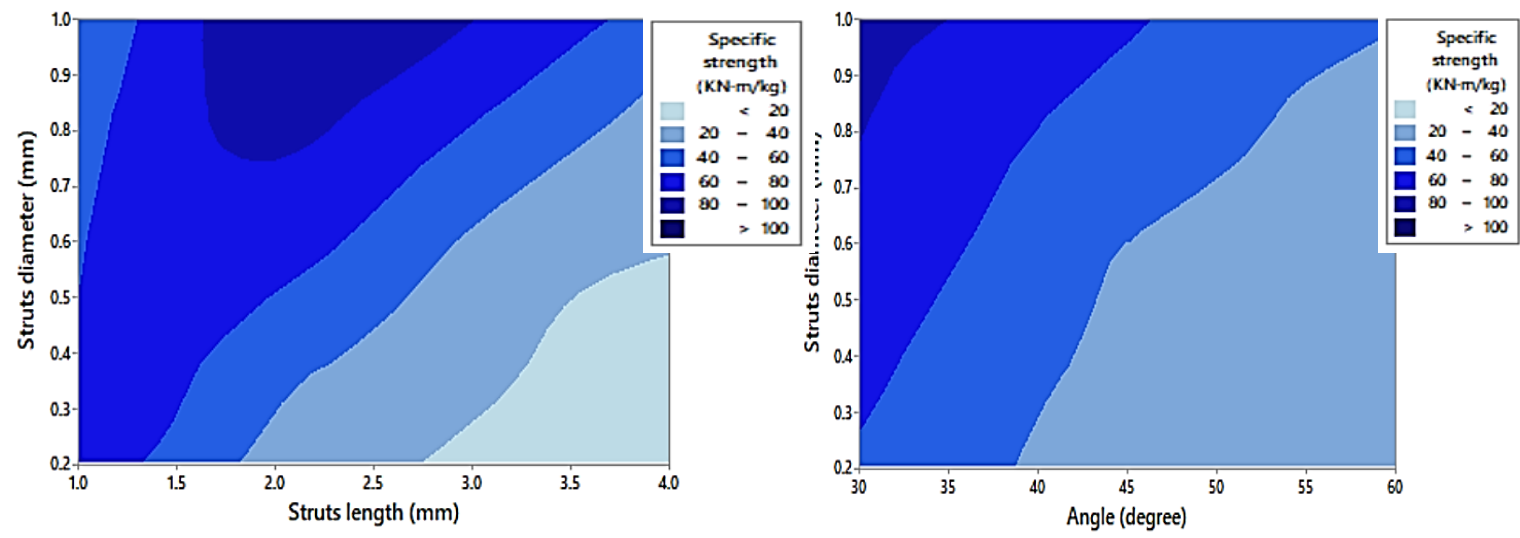

Figure 12: The predicted contours distribution of the specific strength with respect to the lattice struts diameter, length, and angle.

\section{Discussions}

The results showed that the mechanical behaviour of AM cellular structures could be predicted by controlling the cell geometry using deep learning approaches. The methodology presented in this study can be applied for wide range cellular structures regardless to the cell sizes, 
complexity, and materials used as DLNN can be implemented to develop an input-output algorithm where the input parameters are cell geometry while the output mechanical properties are the obtained mechanical characterization. Hence, deep learning was introduced to build predictive mechanical properties model of Ti-64 cellular structures using a limited number of samples with an accuracy better than conventional statistical methods. The correlations between cell geometry and ultimate strength, elastic modulus and specific strength were obtained combining the optimized DLNN model and measured results. The strength and the elastic modulus of the cellular structures can be custom-made for biomedical and aerospace applications. For example, biomedical implants can be tailored made using our approach to achieve a high specific strength with elastic modulus similar to human tissue and hence avoid stress-shielding problem of biomedical implants. The computational cost of the introduced DLNN approach was less than 5 min for the training process. Furthermore, the approach showed it can be implemented as an alternative technique of DoE in the context of process or design optimisation since DLNN mean error was less than the DOE one. Thus, we proposed an efficient algorithm of additively manufactured cellular structures, while demonstrating the approach potential in predicting and optimising designs with geometrical parameters as the diamond cell design is used as a proof-of-concept as well as a qualitative comparison with other approaches. Furthermore, the concept of using additive manufacturing and machine learning to design high performance structures can include a wide range of materials and processes to tailor any interesting property such as optical, mechanical, electric, or thermal.

\section{Conclusions}

Results showed that the DLNN model can accurately predict compression properties of cellular materials and generate distribution model that lead to stronger and controlled stiffness structures. The discussed results lead to the following conclusions:

- The trained DLNN model showed the highest performance when compared to deep neural network (DNN), shallow neural network (SNN) and design of experiment (DoE).

- There is an increase in the compression strength as the strut diameter increases and the strut length decreases. In addition, the elastic modulus increases with the increase of the strut diameter and strut length.

- The specific strength of the samples increases as the dimeter increases while it 
decreases with the increase of the strut length and strut angle.

The trained DLNN model showed that it could predict the occurrence of porosity level with an accuracy of $5.26 \%, 14.60 \%$, and $9.39 \%$ for the ultimate strength, elastic modulus, and specific strength. These results show that the developed DLNN models can be considered as promising tools in learning measurement from few measurement data to make high-fidelity performance predictions. The approach developed in this paper can be also be extended to other cellular types.

\subsection{Experimental Section}

The material used in the fabrication of the lattice structures was Ti-64. The material is lightweight with superior mechanical properties, which makes it suitable for aerospace and biomedical applications. Laser powder bed fusion (LPBF) was used to fabricate the proposed lattice matrix. Ti6Al4V, the powder size range of $25-50 \mu \mathrm{m}$ was supplied by (TLS Technik $\mathrm{GmbH}$, Germany). A Concept Laser M2 LPBF machine is used, which consists of Nd:YAG laser of $1075 \mathrm{~nm}$ wavelength, and a beam spot of $50 \mu \mathrm{m}$. The samples were produced using standard process parameters of Ti6Al4V with laser power of $200 \mathrm{~W}$, a scanning speed of 1200 $\mathrm{mm} / \mathrm{s}$, and layer thickness of 20 micron for compression testing. The samples were produced on a Ti-6Al-4V plate and under Argon control down to $\mathrm{O}_{2}<100 \mathrm{ppm}$. Prior to the compression testing, the samples were sonicated in an acetone bath for 5 minutes to remove a trapped powders and contaminants. The density of the samples was measured using Archimedes approach with a Mettler-Toledo densitometer. Compression testing were carried out at room temperature using Zwick/Roell system on the fabricated samples. Specimens with $5 \times 5 \times 8$ unit cells were compressed along the $\mathrm{Z}$ (build direction). The tests were carried out under a contact speed of $0.1 \mathrm{~mm} / \mathrm{min}$. The compression maximum strengths are expressed by using the nominal engineering strength which equivalent to the maximum force divided by the cross sectional area of the fabricated samples). This way reflects the load bearing capacity of the samples. Specific strength was calculated by dividing the maximum compression strength of the samples by its calculated density. In addition, the Young's modulus of the samples was calculating the nominal strength by the strain. 


\section{References}

[1] O. Guillame-Gentil, O. Semenov, A.S. Roca, T. Groth, R. Zahn, J. Vörös, M. ZenobiWong, Engineering the extracellular environment: Strategies for building 2D and 3D cellular structures, Advanced Materials 22(48) (2010) 5443-5462.

[2] X.P. Tan, Y.J. Tan, C.S.L. Chow, S.B. Tor, W.Y. Yeong, Metallic powder-bed based 3D printing of cellular scaffolds for orthopaedic implants: A state-of-the-art review on manufacturing, topological design, mechanical properties and biocompatibility, Materials Science and Engineering C 76 (2017) 1328-1343.

[3] L. Yang, O. Harrysson, D. Cormier, H. West, H. Gong, B. Stucker, Additive

Manufacturing of Metal Cellular Structures: Design and Fabrication, JOM 67(3) (2015) 608615.

[4] A. Delgoshaei, M.K.A.M. Ariffin, Z. Leman, B.T.H.T.B. Baharudin, C. Gomes, Review of evolution of cellular manufacturing system's approaches: Material transferring models, International Journal of Precision Engineering and Manufacturing 17(1) (2016) 131-149. [5] A. Sabouri, A.K. Yetisen, R. Sadigzade, H. Hassanin, K. Essa, H. Butt, ThreeDimensional Microstructured Lattices for Oil Sensing, Energy \& Fuels 31(3) (2017) 25242529.

[6] R. Felzmann, S. Gruber, G. Mitteramskogler, P. Tesavibul, A.R. Boccaccini, R. Liska, J. Stampfl, Lithography-based additive manufacturing of cellular ceramic structures, Advanced Engineering Materials 14(12) (2012) 1052-1058.

[7] O. Al-Ketan, A. Soliman, A.M. AlQubaisi, R.K. Abu Al-Rub, Nature-Inspired Lightweight Cellular Co-Continuous Composites with Architected Periodic Gyroidal Structures, Advanced Engineering Materials 20(2) (2018).

[8] C. Qiu, N.J.E. Adkins, H. Hassanin, M.M. Attallah, K. Essa, In-situ shelling via selective laser melting: Modelling and microstructural characterisation, Materials \& Design 87 (2015) 845-853.

[9] K. Essa, F. Modica, M. Imbaby, M.A. El-Sayed, A. ElShaer, K. Jiang, H. Hassanin, Manufacturing of metallic micro-components using hybrid soft lithography and microelectrical discharge machining, The International Journal of Advanced Manufacturing Technology 91(1) (2017) 445-452.

[10] H. Klippstein, H. Hassanin, A. Diaz De Cerio Sanchez, Y. Zweiri, L. Seneviratne, Additive Manufacturing of Porous Structures for Unmanned Aerial Vehicles Applications, Advanced Engineering Materials 20(9) (2018) 1800290.

[11] M. Suard, P. Lhuissier, R. Dendievel, J.J. Blandin, F. Vignat, F. Villeneuve, Towards stiffness prediction of cellular structures made by electron beam melting (EBM), Powder Metallurgy 57(3) (2014) 190-195.

[12] S.P. Soe, P. Martin, M. Jones, M. Robinson, P. Theobald, Feasibility of optimising bicycle helmet design safety through the use of additive manufactured TPE cellular structures, International Journal of Advanced Manufacturing Technology 79(9-12) (2015) 1975-1982.

[13] E. Sheydaeian, Z. Fishman, M. Vlasea, E. Toyserkani, On the effect of throughout layer thickness variation on properties of additively manufactured cellular titanium structures, Additive Manufacturing 18 (2017) 40-47.

[14] C. Qiu, S. Yue, N.J.E. Adkins, M. Ward, H. Hassanin, P.D. Lee, P.J. Withers, M.M. Attallah, Influence of processing conditions on strut structure and compressive properties of cellular lattice structures fabricated by selective laser melting, Materials Science and Engineering A 628 (2015) 188-197. 
[15] H. Salem, L.N. Carter, M.M. Attallah, H.G. Salem, Influence of processing parameters on internal porosity and types of defects formed in Ti6Al4V lattice structure fabricated by selective laser melting, Materials Science and Engineering: A 767 (2019) 138387.

[16] S. Li, H. Hassanin, M.M. Attallah, N.J.E. Adkins, K. Essa, The development of TiNibased negative Poisson's ratio structure using selective laser melting, Acta Materialia 105 (2016) 75-83.

[17] C. Yan, L. Hao, A. Hussein, D. Raymont, Evaluations of cellular lattice structures manufactured using selective laser melting, International Journal of Machine Tools and Manufacture 62 (2012) 32-38.

[18] M. Alzahrani, S.K. Choi, D.W. Rosen, Design of truss-like cellular structures using relative density mapping method, Materials and Design 85 (2015) 349-360.

[19] J. Robbins, S.J. Owen, B.W. Clark, T.E. Voth, An efficient and scalable approach for generating topologically optimized cellular structures for additive manufacturing, Additive Manufacturing 12 (2016) 296-304.

[20] D.J. Brackett, I.A. Ashcroft, R.D. Wildman, R.J.M. Hague, An error diffusion based method to generate functionally graded cellular structures, Computers and Structures 138 (2014) 102-111.

[21] X. Qi, G. Chen, Y. Li, X. Cheng, C. Li, Applying Neural-Network-Based Machine Learning to Additive Manufacturing: Current Applications, Challenges, and Future Perspectives, Engineering 5(4) (2019) 721-729.

[22] Y. Bengio, P. Lamblin, D. Popovici, H. Larochelle, Greedy layer-wise training of deep networks, Proceedings of the 19th International Conference on Neural Information Processing Systems, MIT Press, Canada, 2006, pp. 153-160.

[23] G.E. Hinton, R.R. Salakhutdinov, Reducing the Dimensionality of Data with Neural Networks, Science 313(5786) (2006) 504-507.

[24] G.E. Hinton, S. Osindero, Y.-W. Teh, A Fast Learning Algorithm for Deep Belief Nets, Neural Computation 18(7) (2006) 1527-1554.

[25] G.X. Gu, C.-T. Chen, D.J. Richmond, M.J. Buehler, Bioinspired hierarchical composite design using machine learning: simulation, additive manufacturing, and experiment,

Materials Horizons 5(5) (2018) 939-945.

[26] X.Z. Zhang, M. Leary, H.P. Tang, T. Song, M. Qian, Selective electron beam manufactured Ti-6Al-4V lattice structures for orthopedic implant applications: Current status and outstanding challenges, Current Opinion in Solid State and Materials Science 22(3) (2018) 75-99.

[27] E. Yang, M. Leary, B. Lozanovski, D. Downing, M. Mazur, A. Sarker, A. Khorasani, A. Jones, T. Maconachie, S. Bateman, M. Easton, M. Qian, P. Choong, M. Brandt, Effect of geometry on the mechanical properties of Ti-6Al-4V Gyroid structures fabricated via SLM: A numerical study, Materials \& Design 184 (2019) 108165.

[28] F. Tamburrino, S. Graziosi, M. Bordegoni, The design process of additively manufactured mesoscale lattice structures: A review, Journal of Computing and Information Science in Engineering 18(4) (2018).

[29] K. Essa, H. Hassanin, M.M. Attallah, N.J. Adkins, A.J. Musker, G.T. Roberts, N. Tenev, M. Smith, Development and testing of an additively manufactured monolithic catalyst bed for HTP thruster applications, Applied Catalysis A: General 542 (2017) 125-135.

[30] J. Schmidhuber, Deep learning in neural networks: An overview, Neural Networks 61 (2015) 85-117.

[31] J. Brownlee, Machine Learning Algorithms from Scratch: With Python, Jason Brownlee2017. 
